\title{
4-Hydroxyisoleucine ameliorates an insulin resistant-like state in 3T3-LI adipocytes by regulating TACE/TIMP3 expression
}

This article was published in the following Dove Press journal:

Drug Design, Development and Therapy

20 October 2015

Number of times this article has been viewed

\section{Feng Gao'}

Wen $\mathrm{Du}^{1,4}$

Mohammad Ishraq Zafar'

Raja Adeel Shafqat ${ }^{2}$

Liumeng Jian'

Qin Cai'

Furong $\mathrm{Lu}^{3}$

'Department of Endocrinology, Union Hospital, ${ }^{2}$ Department of Medicine, Tongji Hospital, ${ }^{3}$ Department of Integrated Traditional Chinese and Western Medicine, Union Hospital, Tongji Medical College, Huazhong University of Science and Technology, Wuhan, Hubei, ${ }^{4} \mathrm{Chengdu}$ First People's Hospital, Sichuan, People's Republic of China
Correspondence: Furong Lu Department of Integrated Traditional Chinese and Western Medicine, Union Hospital, Tongji Medical College, Huazhong University of Science and Technology, 1277 Jiefang Avenue, Wuhan, Hubei 430022, People's Republic of China Email furonglulove@।63.com
Background: Obesity-associated insulin resistance (IR) is highly correlated with soluble tumor necrosis factor- $\alpha$ (sTNF- $\alpha$ ), which is released from transmembranous TNF- $\alpha$ by TNF- $\alpha$ converting enzyme (TACE). In vivo, TACE activity is suppressed by tissue inhibitor of metalloproteinase 3 (TIMP3). Agents that can interact with TACE/TIMP3 to improve obesity-related IR would be highly valuable. In the current study, we assessed whether $(2 S, 3 R, 4 S)$-4-hydroxyisoleucine (4-HIL) could modulate TACE/TIMP3 and ameliorate an obesity-induced IR-like state in 3T3-L1 adipocytes.

Materials and methods: 3T3-L1 adipocytes were incubated in the presence of $25 \mathrm{mM}$ glucose and $0.6 \mathrm{nM}$ insulin to induce an IR-like state, and were then treated with different concentrations of 4-HIL or $10 \mu \mathrm{M}$ pioglitazone (positive control). The glucose uptake rate was determined using the 2-deoxy-[ $\left[{ }^{3} \mathrm{H}\right]$-D-glucose method, and the levels of sTNF- $\alpha$ in the cell supernatant were determined using ELISA. The protein expression of TACE, TIMP3, and insulin signaling-related molecules was measured using western blotting.

Results: Exposure to high glucose and insulin for 18 hours increased the levels of sTNF- $\alpha$ in the cell supernatant. The phosphorylation of insulin receptor substrate-1 (IRS-1) Ser ${ }^{307}$ and Akt Ser ${ }^{473}$ was increased, whereas the protein expression of IRS-1, Akt, and glucose transporter-4 was decreased. The insulin-induced glucose uptake was reduced by $67 \%$ in 3T3-L1 adipocytes, which indicated the presence of an IR-like state. The above indexes, which demonstrated the successful induction of an IR-like state, were reversed by 4-HIL in a dose-dependent manner by downregulating and upregulating the protein expression of TACE and TIMP3 proteins, respectively.

Conclusion: 4-HIL improved an obesity-associated IR-like state in 3T3-L1 adipocytes by targeting TACE/TIMP3 and the insulin signaling pathway.

Keywords: 4-hydroxyisoleucine, obesity-related insulin resistance, soluble TNF- $\alpha, T A C E$, TIMP3, insulin signaling, glucose uptake, Fenugreek seed

\section{Introduction}

An increasing amount of epidemiological data suggest that obesity plays a vital role in the development of many life-threatening diseases, and especially insulin resistance (IR). ${ }^{1}$ IR is a chronic low-grade inflammatory disease. ${ }^{2,3}$ Inflammatory cytokines, such as tumor necrosis factor- $\alpha$ (TNF- $\alpha$ ), interleukin-6 (IL-6), and C-reactive protein (CRP), are highly correlated with IR. Among these, TNF- $\alpha$ has drawn considerable attention because of its various biological effects..$^{4-8}$

TNF- $\alpha$ is mainly released by the mononuclear phagocyte system. It is synthesized as a transmembrane 26-kDa precursor transmembrane tumor necrosis factor- $\alpha$ (mTNF- $\alpha$ ), which is cleaved into the secreted $17-\mathrm{kDa}$ form (sTNF- $\alpha$ ) by TNF- $\alpha$ converting 
enzyme $(T A C E)$ at $76 \mathrm{Ala}-77 \mathrm{Vla}$ in humans. ${ }^{9}$ mTNF- $\alpha$ and sTNF- $\alpha$ have different structures and functions. It was reported that sTNF- $\alpha$ could induce systemic IR by interfering with insulin signal transduction. ${ }^{3,10}$ sTNF- $\alpha$ reduces insulin sensitivity by interfering with the tyrosine phosphorylation of insulin receptor substrate (IRS) ${ }^{11}$ and downregulating glucose transporter-4 (GLUT4) expression. ${ }^{12}$ Furthermore, sTNF- $\alpha$ plays an important role in promoting adipocyte decomposition and free fatty acid release, thereby aggravating IR. ${ }^{5}$ In contrast, mTNF- $\alpha$ has much lesser effects on the insulin signaling pathway and lipid metabolism. Xu et al reported that the specific expression of mTNF- $\alpha$ resulted in local, but not systemic, IR and reduced the amount of adipose tissue in mTNF $1-9 \mathrm{~K} 11 \mathrm{E}$ transgenic mice. ${ }^{13}$ Additional studies indicated that mTNF- $\alpha$ promoted the formation of adipose tissue, but did not affect insulin-induced glucose metabolism in high-fat diet (HFD)-fed mice. ${ }^{14}$ Therefore, sTNF- $\alpha$, which is markedly increased in obesity, is one of the most important inflammatory cytokines that contribute to obesity-induced IR.

$T A C E$, also named a disintegrin and metalloproteinase 17 (ADAM17) and CD156b, is the main TNF- $\alpha$ lyase in vivo. It is secreted in a zymogen form, and belongs to the ADAM family. Tissue inhibitor of metalloproteinase 3 (TIMP3) is now recognized as a natural inhibitor of $T A C E .{ }^{15}$ Extensive research in recent years has suggested that the inhibition of $T A C E$ might play a key role in ameliorating obesity or IR. Fiorentino et al reported that either increased TACE expression and activity or decreased TIMP3 expression resulted in IR and hepatosteatosis in HFD-fed mice. ${ }^{16}$ Therefore, the TACE/TIMP3 system is emerging as a key player in IR.

$(2 S, 3 R, 4 S)-4$-Hydroxyisoleucine (4-HIL) is a plantderived structural agent that can lower blood sugar and also correct dyslipidemia. Since it was extracted from the seeds of Trigonella foenum-graecum in the 1970s, 4-HIL has aroused widespread interest. Based on in vitro and in vivo data, Broca et al suggested that 4-HIL exerts glucose-dependent insulinotropic effects and decreases fasting blood glucose. They also indicated that 4-HIL could improve glucose tolerance in type 2 diabetes mellitus (T2DM) rats. ${ }^{17,18}$ According to Singh et al, 4-HIL stimulated glucose uptake in peripheral tissues; therefore, it plays a role in preventing the aggravation of diabetes. ${ }^{19}$ Moreover, 4-HIL reduced triglyceride and total cholesterol levels and increased the high-density lipoprotein cholesterol/total cholesterol ratio in a dyslipidemic hamster model. ${ }^{20}$

Our previous study investigated the effects of 4-HIL on a high glucose-induced IR-like state in 3T3-L1 adipocytes from mice, and found that 4-HIL could remarkably improve the high glucose-induced IR-like state in 3T3-L1 adipocytes. ${ }^{21}$ The present study aimed to investigate the effects of 4-HIL on an obesity-induced IR-like state and explore the underlying molecular mechanisms behind these effects.

\section{Materials and methods Reagents}

4-HIL ( $>98 \%$ purity) was obtained from Sigma-Aldrich (St Louis, MO, USA), dissolved in triple-distilled water at a concentration of $100 \mathrm{mM}$, stored at $-20^{\circ} \mathrm{C}$, and then further diluted in cell culture medium immediately before use.

Antibodies against TACE and TIMP3 were purchased from Abcam (Cambridge, UK). Antibodies against IRS-1, IRS-2, phospho-IRS-1 Ser ${ }^{307}$, phospho-IRS-1 Ser ${ }^{318}$, Akt, and phospho-Akt Ser ${ }^{473}$ were obtained from Cell Signaling Technology (Danvers, MA, USA). Anti-GLUT4 antibody was obtained from Santa Cruz Biotechnology (Santa Cruz, CA, USA). Goat anti-mouse HRP-conjugated and goat anti-rabbit HRP-conjugated antibodies were also purchased from Cell Signaling Technology. The mouse TNF- $\alpha$ ELISA kit was obtained from Neobioscience (Shenzhen, People's Republic of China).

Penicillin, streptomycin, Dulbecco's Modified Eagle's Medium (DMEM), and fetal bovine serum were obtained from GIBCO BRL Life Technologies (Grand Island, NY, USA). Insulin, 3-isobutyl-1-methylxanthine, dexamethasone, oil red $\mathrm{O}$, and 2-deoxy- $\left[{ }^{3} \mathrm{H}\right]$-D-glucose (2-DOG) were purchased from Sigma-Aldrich. Protease and phosphatase inhibitor cocktails were purchased from Servicebio (Wuhan Goodbio Technology CO., Ltd, Wuhan, People's Republic of China). The other reagents used in this study were obtained from Sigma-Aldrich.

\section{T3-LI cell culture and differentiation induction}

3T3-L1 preadipocytes were purchased from American Type Culture Collection (Manassas, VA, USA), and were grown in DMEM supplemented with $10 \%$ fetal bovine serum (complete medium; CM) and antibiotics $(100 \mathrm{U} / \mathrm{mL}$ penicillin and $100 \mu \mathrm{g} / \mathrm{mL}$ streptomycin) in a $37^{\circ} \mathrm{C}$ incubator containing 5\% $\mathrm{CO}_{2}$. Confluent 3T3-L1 cells were differentiated in CM supplemented with $25 \mathrm{mM}$ glucose, $10 \mu \mathrm{g} / \mathrm{mL}$ insulin, $0.5 \mathrm{mM}$ 3-isobutyl-1-methylxanthine, and $1 \mu \mathrm{M}$ dexamethasone. On day 2 of differentiation, the adipogenic cocktail was changed to $\mathrm{CM}$ with $10 \mu \mathrm{g} / \mathrm{mL}$ insulin. The cells were cultivated in CM until day 4 and were then refed 
every other day. ${ }^{22,23}$ Oil red O staining on days 8-12 revealed that $>90 \%$ of the induced cells had acquired the adipocyte morphology. For quantification oil red $\mathrm{O}$ was eluted using $100 \%$ isopropanol.

\section{Methyl thiazolyl tetrazolium assays}

Methyl thiazolyl tetrazolium (MTT) assays were performed to assess cell viability, as described previously. ${ }^{24}$ Briefly, 3T3-L1 adipocytes in the logarithmic growth phase were collected and adjusted to the appropriate concentration. Then, $100 \mu \mathrm{L}$ of cell suspension $\left(10^{5} / \mathrm{mL}\right)$ was seeded in triplicate into 96-well plates in the presence or absence of the indicated concentrations of 4-HIL for the specified time periods. After incubation, $20 \mu \mathrm{L}$ MTT solution ( $5 \mathrm{mg} / \mathrm{mL}$ MTT) was added to each well, and the plates were incubated for an additional 4 hours at $37^{\circ} \mathrm{C}$. Next, $150 \mu \mathrm{L}$ dimethyl sulfoxide was added to each well, and the plates were shaken at a low speed for 10 minutes. Finally, the cell viability was determined colorimetrically at $492 \mathrm{~nm}$ using a microplate reader (Thermo Electron Corp., Waltham, MA, USA).

\section{2-DOG uptake}

Mature adipocytes were transferred into serum-free DMEM containing $2 \mathrm{~g} / \mathrm{L}$ bovine serum albumin (BSA) in six-well plates for 12 hours, followed by $10 \mathrm{~g} / \mathrm{L}$ BSA with $0.6 \mathrm{nM}$ insulin for 18 hours. They were then co-cultured with different concentrations of 4-HIL for another 24 hours. ${ }^{25}$ Glucose uptake was measured in the treated 3T3-L1 cells as follows. Differentiated 3T3-L1 cells were washed three times in warm Krebs-Ringer HEPES (KRH) buffer, and then treated with $100 \mathrm{nM}$ insulin; $10 \mu \mathrm{M}$ cytochalasin $\mathrm{B}$ was added to some wells as a control. Cells were cultivated for 30 minutes at $37^{\circ} \mathrm{C}$, and then $0.5 \mu \mathrm{Ci} / \mathrm{mL} 2-D O G$ was added to each well for an additional 10 minutes. The incubation was terminated by placing cells on ice and washing with precooled KRH buffer. Finally, cells were lysed in $0.1 \%$ sodium dodecyl sulfate, and the glucose uptake rate was determined using scintillation counting. ${ }^{23}$

\section{ELISA}

A sandwich ELISA was adopted to detect mouse sTNF- $\alpha$ according to the manufacturer's instructions. Cell culture supernatants were centrifuged for 20 minutes at 2,000 rpm before assaying. After the reaction had been terminated, the color change was detected spectrophotometrically at a wavelength of $450 \mathrm{~nm}$. The concentration of TNF- $\alpha$ in the samples was then determined by comparing the optical density (OD) of the samples with standard curves.

\section{Western blotting}

Fully differentiated 3T3-L1 adipocytes seeded in sixwell plates were incubated under indicated conditions for 24 hours. They were then washed three times in precooled phosphate buffered saline, and lysed in radioimmuno precipitation assay lysis buffer supplemented with protease inhibitors and phosphatase inhibitors. The protein concentrations were determined using a bicinchoninic acid (BCA) protein assay reagent kit (Pierce, Rockford, IL, USA). Proteins $(50 \mu \mathrm{g})$ from each group were separated on sodium dodecyl sulfate polyacrylamide gel electrophoresis gels, and electro transferred to polyvinylidene difluoride membranes. Thereafter, the membranes were blocked using $5 \%$ low-fat powdered milk in Tris-buffered saline $/ 0.1 \%$ Tween-20 (TBST) or with 5\% BSA in TBST for 1 or 2 hours at room temperature.

After blocking, the membranes were incubated overnight at $4^{\circ} \mathrm{C}$ with primary antibodies against TACE, TIMP3, IRS-1, IRS-2, phospho-IRS-1 Ser ${ }^{307}$, phospho-IRS-1 Ser ${ }^{318}$, Akt, phospho-Akt Ser ${ }^{473}$, GLUT4, and $\beta$-actin. The membranes were washed three times each for 1 minute using TBST, and were then incubated with HRP-conjugated secondary antibodies for 1 hour with gentle agitation at room temperature. After washing, the immunoreactive bands were measured using an enhanced chemiluminescence kit and a chemiluminescence system (Bio-Rad, Hercules, CA, USA). ${ }^{26}$

\section{Statistical analysis}

Data are presented as means \pm SE. Statistical and graphical analyses were performed using SPSS version 19.0 software. The experimental results were analyzed for statistical significance using analysis of variance where appropriate; $P<0.05$ was considered to indicate statistical significance.

\section{Results}

\section{4-HIL increases glucose uptake in IR-like 3T3-LI adipocytes}

To investigate whether 4-HIL could enhance glucose uptake, fully differentiated 3T3-L1 adipocytes were employed. First, an IR-like cell model was induced using high glucose and insulin, and the changes in 2-DOG uptake rate were measured. Data revealed that stimulation with $25 \mathrm{mM}$ glucose and $0.6 \mathrm{nM}$ insulin for 18 hours reduced the 2-DOG uptake rate of 3T3-L1 adipocytes by $67 \%$, which indicated the presence of an IR-like state. ${ }^{23}$ However, the addition of 4-HIL for 24 hours corrected the IR-like state, with a peak glucose uptake at $20 \mu \mathrm{mol} / \mathrm{L} 4-\mathrm{HIL}$ (Figure 1). 


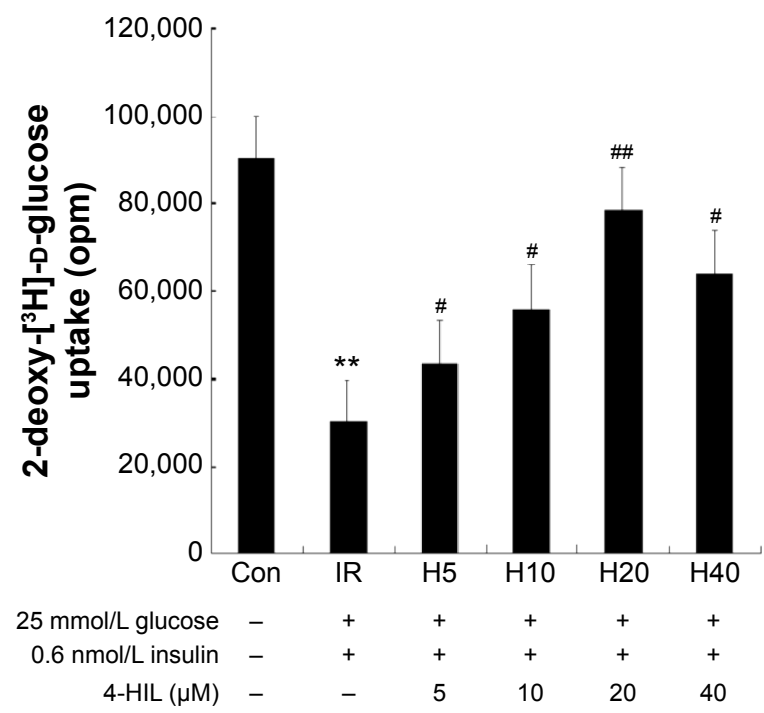

Figure I The effects of high glucose, insulin, and different concentrations of 4-HIL on insulin-induced 2-DOG uptake in 3T3-LI adipocytes.

Notes: Another group was treated with only $10 \mu \mathrm{M}$ cytochalasin B (data not shown) to measure the nonspecific uptake of 2-DOG (background). Each value in the figure was subtracted from the background value. Data represent the mean \pm SE of three duplicate experiments $(n=3)$. ${ }^{* * P}<0.0$ I vs control; ${ }^{\#} P<0.05,{ }^{\# P}<0.0$ I vs the IR group.

Abbreviations: 4-HIL, (2S,3R,4S)-4-hydroxyisoleucine; 2-DOG, 2-deoxy-[3H]-Dglucose; IR, insulin resistance; con, control; SE, standard error.

\section{4-HIL inhibits the over-secretion of sTNF- $\alpha$ in IR-like 3T3-LI adipocytes}

Adipose tissue is an endocrine organ that can produce a number of cytokines, including TNF- $\alpha$, leptin, resistin, and IL-6, which are all over expressed during obesity and contribute to IR. ${ }^{27}$ To determine whether 4-HIL could alleviate the inflammatory state of adipocytes by reducing the over-secretion of proinflammatory factors, we measured TNF- $\alpha$ levels in supernatants isolated from IR-like and 4-HIL-treated 3T3-L1 adipocytes. Surprisingly, 4-HIL decreased sTNF- $\alpha$ levels in 3T3-L1 cells in a dose-dependent manner (Figure 2).

\section{4-HIL downregulates TACE and upregulates TIMP3 expression simultaneously in IR-like 3T3-LI adipocytes}

Because TACE and TIMP 3 are the key regulators of sTNF- $\alpha$ levels, ${ }^{28}$ we next investigated the effects of 4-HIL on TACE and TIMP3 protein expression in 3T3-L1 adipocytes. Data revealed that $T A C E$ levels were decreased in a dosedependent manner after treatment with 4-HIL (Figure 3A, upper row). Quantification of the western blots demonstrated that the expression of TACE in the H20 group was decreased significantly by $46 \%$ compared with the IR-like group (Figure 3A, lower row). Although the mechanism by

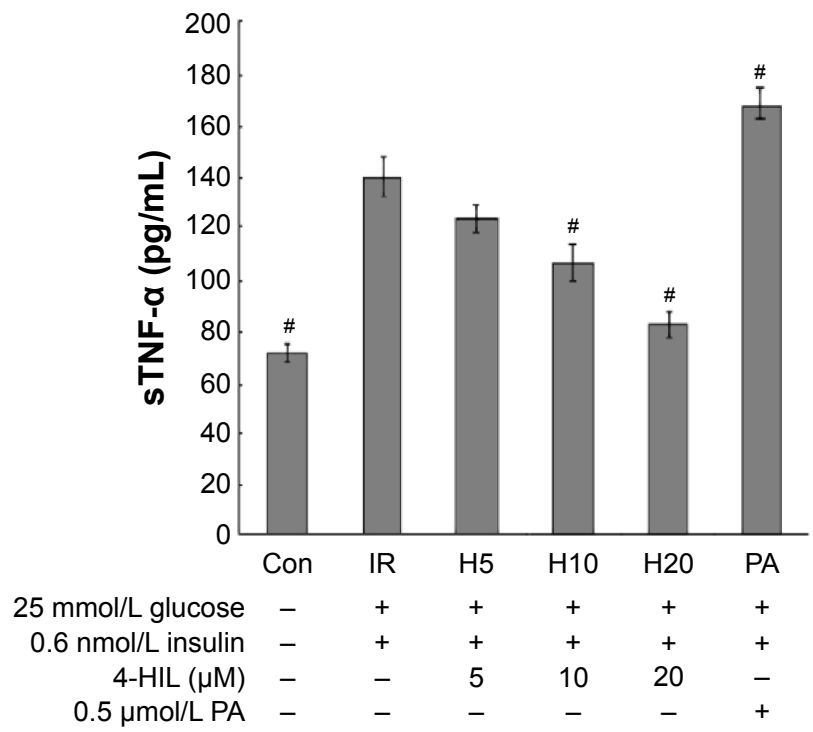

Figure 2 The concentration of released sTNF- $\alpha$ in IR-like 3T3-LI adipocytes, as measured using ELISA.

Notes: PA, palmitic acid (TACE activator); used as a positive control. The samples analyzed were cell supernatants, and three replicate wells were assayed per group. Data are presented as the mean \pm SE of three experiments $(n=3) .{ }^{\#}<0.05$ vs the IR-like group.

Abbreviations: sTNF- $\alpha$, soluble tumor necrosis factor- $\alpha$; IR, insulin resistance; TACE, TNF- $\alpha$ converting enzyme; con, control; 4-HIL, (2S,3R,4S)-4-hydroxyisoleucine; $\mathrm{SE}$, standard error.

which $T A C E$ is activated remains unclear, TIMP3, which is a natural competitive inhibitor of $T A C E$, plays an important role in vivo. Therefore, we assessed the protein expression of TIMP3 in 3T3-L1 adipocytes. Compared with the IR-like group, 4-HIL enhanced TIMP3 protein expression in a dosedependent manner (Figure 3A, middle row). Specifically, treatment with $20 \mu \mathrm{M}$ 4-HIL increased TIMP3 expression by $89 \%$ (Figure 3B). Pioglitazone (PIO), a peroxisome proliferator-activated receptor (PPAR)- $\gamma$ agonist, can alleviate TNF- $\alpha$-induced IR by specifically blocking the insulin signaling pathway; ${ }^{29,30}$ therefore, PIO was used as a positive control in this experiment.

\section{The effects of 4-HIL on the insulin signaling pathway in IR-like 3T3-LI adipocytes}

A mechanism by which TNF- $\alpha$ induces IR is by interacting with the insulin signaling pathway. Therefore, we next assessed whether 4-HIL could partially or completely counteract the effects of TNF- $\alpha$ on the insulin receptor and its postreceptor signal transduction pathway. The total protein expression of IRS-1 was low in IR 3T3-L1 adipocytes, but was upregulated by high-dose 4-HIL (H10, H20, and H40 groups), but not low-dose 4-HIL (the H5 group; Figure 4A). Consistent with previous reports, the positive control PIO 

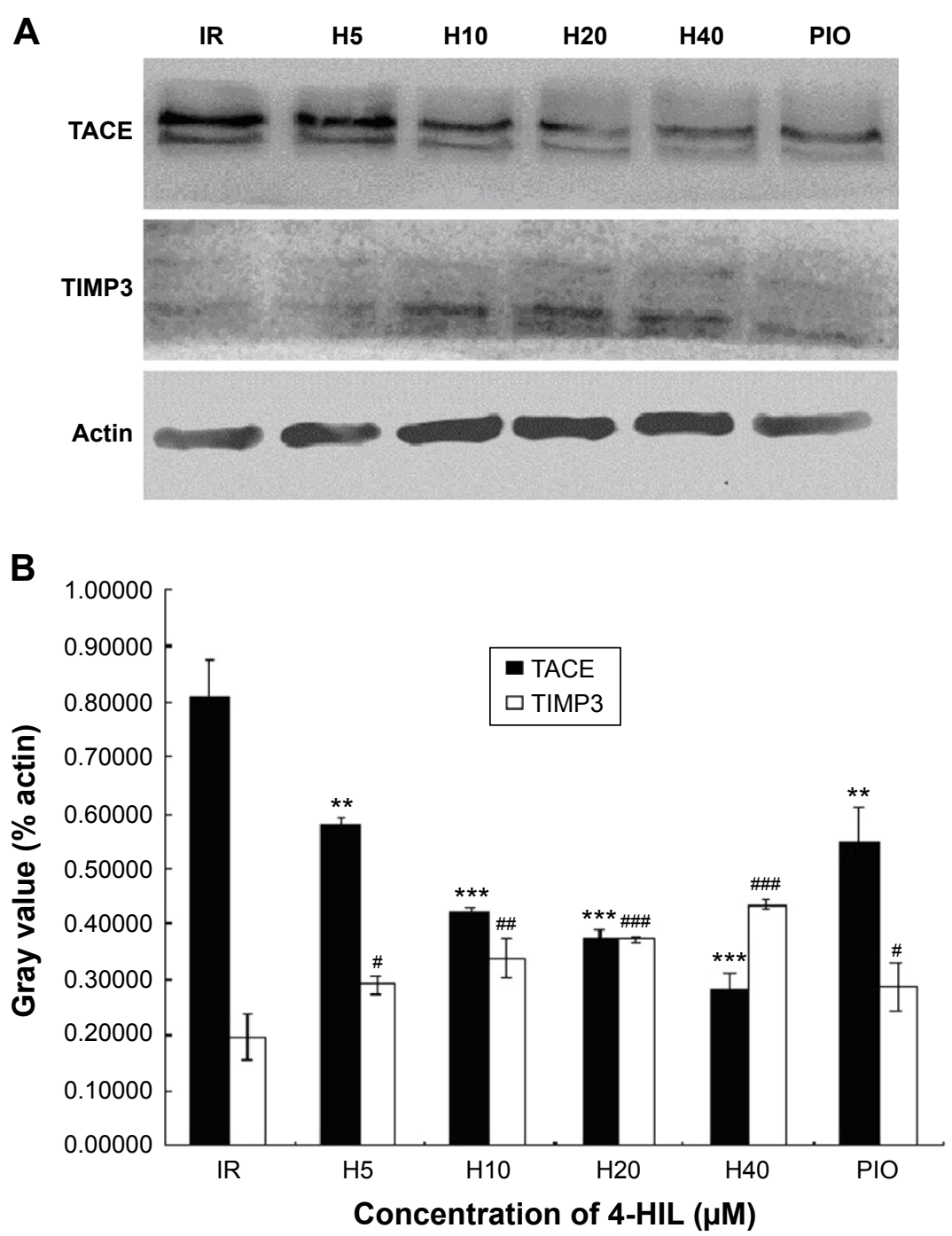

Figure 3 The effect of 4-HIL on the TACE/TIMP3 system in IR 3T3-LI adipocytes.

Notes: Cells were incubated with the indicated concentrations of 4-HIL or $10 \mu \mathrm{M}$ PIO for 24 hours, and then total proteins were extracted and analyzed by immunoblotting for TACE and TIMP3. The same blot was then stripped and reprobed with anti- $\beta$-actin antibody to ensure equal protein loading (A). PIO was used as a positive control. The figure shows a representative gel from one experiment. The values are presented as the mean $\pm S E$ of three independent experiments. $* * P<0.0 \mathrm{I}$, $* * * P<0.00 \mathrm{I}$ vs the IR-like

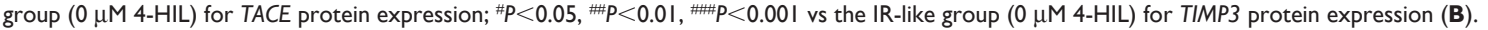

Abbreviations: 4-HIL, (2S,3R,4S)-4-hydroxyisoleucine; TACE, TNF- $\alpha$ converting enzyme; TIMP3, Tissue inhibitor of metalloproteinase 3; IR, insulin resistance; PIO, pioglitazone; SE, standard error.

also increased IRS-1 levels compared with the IR-like group (Figure 4A). We also assessed the phosphorylation of IRS-1 Ser ${ }^{307}$ and IRS-1 Ser $^{318}$, and found that phospho-IRS-1 $\mathrm{Ser}^{307}$ was inhibited by 4-HIL in a dose-dependent manner (Figure 4A). However, 4-HIL did not decrease phosphoIRS-1 Ser ${ }^{318}$ levels significantly.

Because Akt plays a major role in connecting PI-3 kinase to GLUT4 in the insulin postreceptor signaling cascade, we next investigated the protein expression of Akt and phosphoAkt $\mathrm{Ser}^{473}$. There were no statistically significant differences in the expression of total Akt after incubation with 4-HIL. However, phospho-Akt Ser ${ }^{473}$ levels were reduced by 4-HIL in a dose-dependent manner (Figure 4B).
Finally, GLUT4 protein, which is also a downstream molecule in the insulin signaling pathway, was investigated. Data revealed that 4-HIL increased GLUT4 protein expression markedly in a dose-dependent manner; a 13.6-fold increase was observed in the $\mathrm{H} 20$ group compared with the IR group (Figure 4C).

\section{4-HIL does not affect the proliferation or differentiation of 3T3-LI preadipocytes}

To assess the cytotoxicity of 4-HIL, we measured the viability of 3T3-L1 preadipocytes that had been treated with different concentrations of 4-HIL using MTT assays. There was no significant difference in cell viability after 

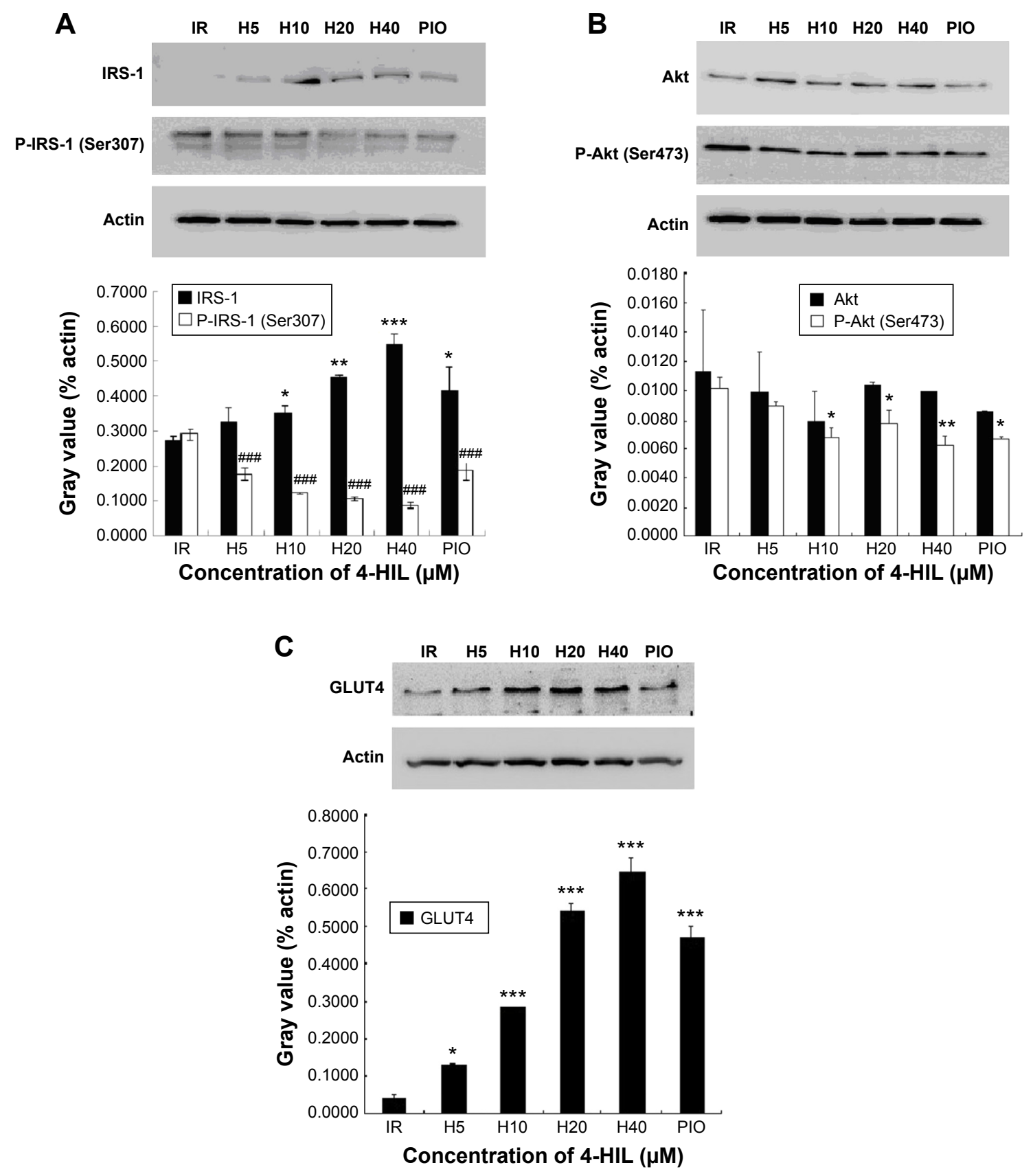

Figure 4 The effect of 4-HIL on the insulin signaling pathway in IR-like 3T3-LI adipocytes.

Notes: The cells were treated with 4-HIL for 24 hours, lysed, and then analyzed using immunoblotting for key proteins involved in the insulin signaling pathway. Representative blots and quantitative bar graphs for the expression of IRS-I and P-IRS-I (Ser307) (A), Akt and P-Akt (Ser473) (B), and GLUT4 (C) in 3T3-LI adipocytes treated with different concentrations of 4-HIL are shown; PIO was used as a positive control. The figure shows a representative gel from one experiment. The values are presented as the mean $\pm S E$ of three independent experiments. $* P<0.05$, $* * P<0.0$ I, $* * * P<0.00$ I vs the IR-like group $(0 \mu M$ 4-HIL $)$ for the expression of IRS-I; ${ }^{\prime \prime} P<0.00$ I vs the IR-like group (0 $\mu$ M 4-HIL). P-IRS-I (Ser307), phospho-IRS-I Ser ${ }^{307}$; P-Akt-I (Ser473), phospho-Akt Ser ${ }^{473}$ for the expression of P-IRS-I (Ser307).

Abbreviations: 4-HIL, (2S,3R,4S)-4-hydroxyisoleucine; PIO, pioglitazone; IR, insulin resistance; IRS-I, insulin receptor substrate-I; GLUT4, glucose transporter-4; SE, standard error.

24 hours exposure to $0-200 \mu \mathrm{M} 4-\mathrm{HIL}$. When we extended the exposure time to 48 hours there were still no detectable cytotoxic effects of 4-HIL, even at a concentration of $200 \mu \mathrm{M}$, which had the lowest survival rate of $89.70 \%$ (Figure 5).

Finally, we investigated whether 4-HIL influence could affect the differentiation of 3T3-L1 preadipocytes. There was almost no difference in the number of lipid droplets or cell morphology before and 8 days after the addition of $20 \mu \mathrm{M}$ or $40 \mu \mathrm{M} 4$-HIL (Figure 6A). To confirm these observations, we eluted oil red O staining by adding $100 \%$ isopropanol to dissolve and quantitatively analyze the lipid droplets. Measuring the absorbance at $490 \mathrm{~nm}$ using a microplate reader revealed no differences in the lipid droplet levels among 


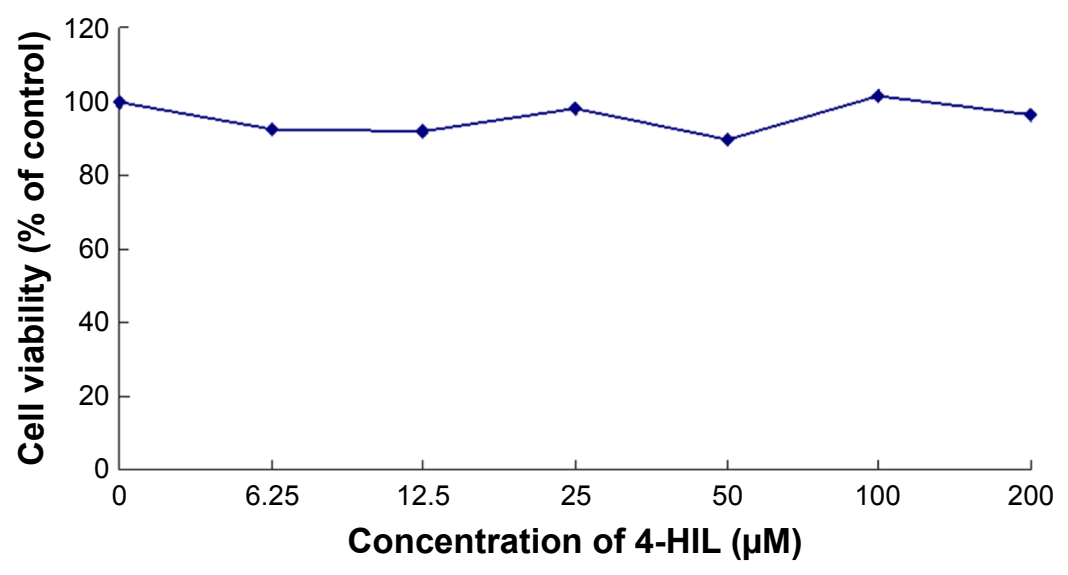

Figure 5 The viability of 3T3-LI preadipocytes after exposure to different concentrations of 4-HIL for 48 hours. Note: Data are presented as the mean \pm SE of three independent experiments. Abbreviations: 4-HIL, (2S,3R,4S)-4-hydroxyisoleucine; SE, standard error.

groups (Figure 6B). This suggests that 4-HIL does not affect the differentiation of 3T3-L1 preadipocytes.

\section{Discussion}

4-HIL is a plant-derived, nonprotein amino acid with glucoselowering and lipid-lowering properties. Previous studies revealed that the mechanism of action of 4-HIL involves inhibiting glucose absorption, stimulating insulin synthesis and secretion, ${ }^{18}$ and activating the insulin signaling pathway. ${ }^{17}$ Broca et $\mathrm{a}^{17}$ suggested that 4 -HIL could improve IR by increasing peripheral glucose utilization and inhibiting hepatic glucose production in Zucker fa/fa rats. 4-HIL was also found to stimulate PI3-K activity in the muscle of diabetic rats. However, the underlying mechanisms of 4-HIL on the insulin signaling pathway have not been studied thoroughly.

The endocrine dysfunction of adipose tissue plays an important role in the pathophysiological progression of obesity to IR and T2DM, and interacts as both a cause and an effect. Meanwhile, IR in adipose tissue can be attributed to many factors. Abundant research has suggested that inflammation and the insulin signaling pathway crosstalk at many levels (Figure 7). ${ }^{31}$ Some kinases, such as c-Jun N-terminal kinase (JNK), IkB kinase (IKK), and mitogen-activated protein kinase (MAPK), are activated by TNF- $\alpha$ and other inflammatory markers, and then promote insulin receptor and IRS phosphorylation at serine and threonine residues rather than tyrosine. This suppresses IRS to subsequently weaken the downstream signaling cascade. ${ }^{32}$ Furthermore, TNF- $\alpha$ decreases insulin-stimulated glucose uptake by inhibiting the production and translocation of GLUT4. ${ }^{12}$ Some studies linked TACE, an activator of sTNF- $\alpha$, to IR. Serino et al noted that TACE haploinsufficiency reduced the homeostatic model assessment-insulin resistance (HOMA-IR) index dramatically in HFD-fed $\mathrm{TACE}^{+/-}$mice. ${ }^{33}$ Similar preliminary studies have been performed in humans. For example, Monroy et al compared the differences in TACE protein and gene expression between subjects with normal glucose tolerance and T2DM patients, and concluded that there were was much higher activity and increased expression of TACE in the muscle of T2DM sufferers compared with normal glucose tolerance subjects. These observations were closely related to IR; ${ }^{34}$ however, the exact mechanism behind these effects was unknown.

Based on the earlier observations, the current study tested the hypothesis that 4-HIL increases insulin-stimulated glucose uptake and thereby improves the IR-like state in 3T3-L1 adipocytes by 1 ) inhibiting TNF- $\alpha$ production by downregulating TACE expression and upregulating TIMP3 expression and 2) inhibiting the phosphorylation of IRS-1 Ser ${ }^{307}$ and Akt $\mathrm{Ser}^{473}$, which are inhibitory molecules in the insulin signaling pathway. We also hypothesized that 4-HIL increases GLUT4 translocation at the cellular membrane (Figure 7). Therefore, we conducted the present study to investigate the effects of 4-HIL on TNF- $\alpha$-induced IR and the underlying molecular mechanisms in 3T3-L1 adipocytes. First, we demonstrated that 4-HIL increased glucose uptake in IR-like 3T3-L1 adipocytes in a dose-dependent manner (Figure 1). We next investigated the potential molecular mechanisms behind this observation. Data revealed that 4-HIL could reduce the over-secretion of sTNF- $\alpha$ (Figure 2). To gain an insight into the anti-TNF- $\alpha$ mechanisms of 4-HIL, we then measured the protein levels of TACE and TIMP3, and found that 4-HIL downregulated and upregulated the expression of TACE and $T I M P 3$, respectively (Figure 3). This subsequently decreased the conversion of mTNF- $\alpha$ to sTNF- $\alpha$, and thereby blocked TNF- $\alpha$ signal transduction. Therefore, one mechanism 

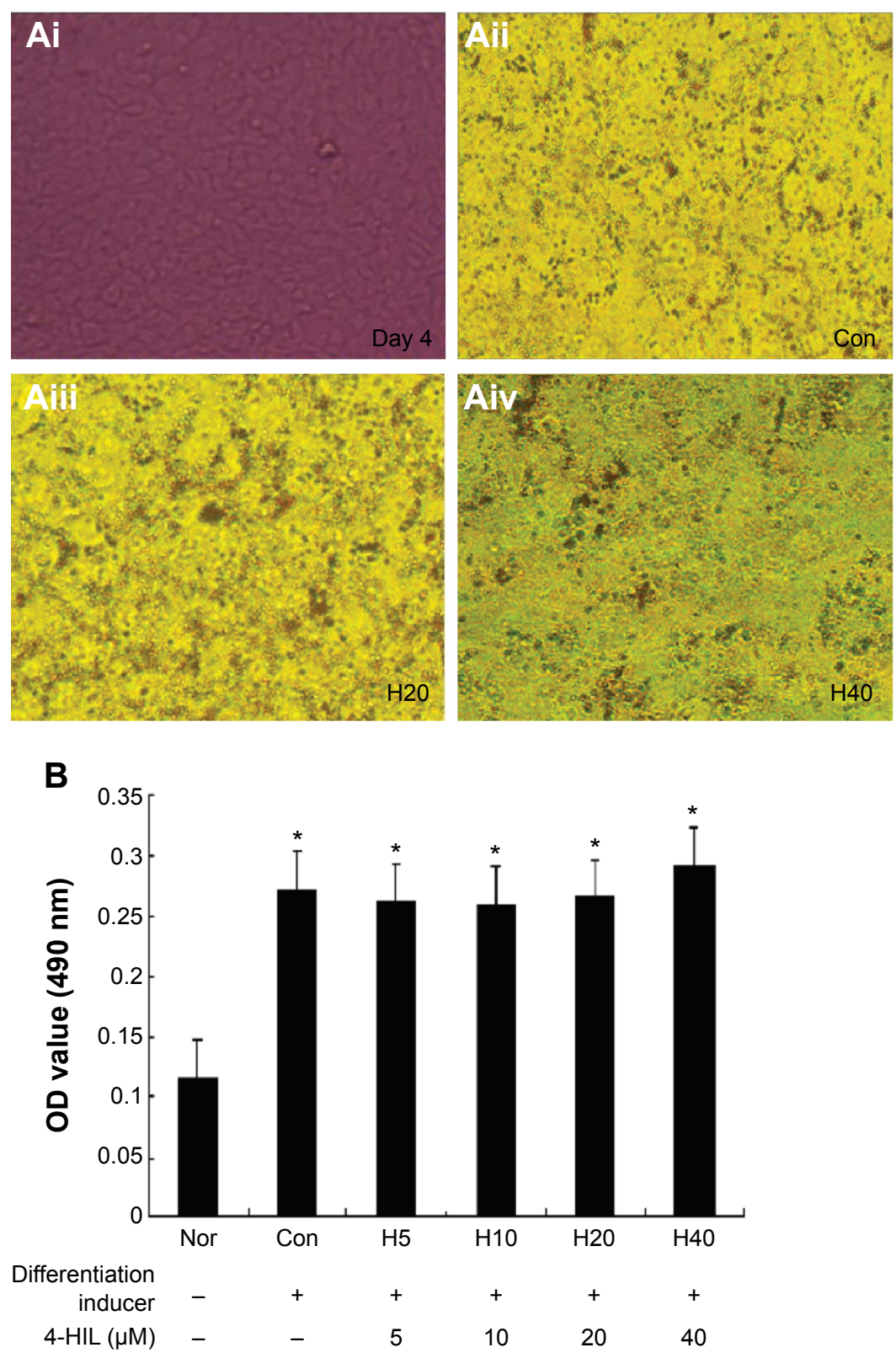

Figure 6 Effects of 4-HIL on the differentiation of 3T3-LI preadipocytes.

Notes: A cell differentiation inducer (adipogenic cocktail) was added on day 0 , and 4-HIL was added on day 4. Cell culture was terminated on day 8 by incubation with a filtered oil red $\mathrm{O}$ solution for 30 minutes at room temperature followed by washing with $70 \%$ ethanol; the solution was then dissolved in DMSO. For quantification, oil red O was eluted by the addition of $100 \%$ isopropanol. Cell morphology on day 4 (Ai, preadipocytes), day 8 (Aii), day 8 with $20 \mu$ M 4 -HIL (Aiii), and day 8 with $40 \mu$ M 4-HIL (Aiv) is shown. The latter three pictures on day 8 reveal that the cells were rounded with intracellular fat droplets; therefore, they were mature or fully differentiated $3 T 3-L I$ adipocytes. (B) Lipid droplet quantification on day 8 . Nor, without the differentiation inducer; Con, with the differentiation inducer. Data are presented as the means \pm SE of three independent experiments. $* P<0.05$ vs the Nor group.

Abbreviations: 4-HIL, (2S,3R,4S)-4-hydroxyisoleucine; DMSO, dimethyl sulfoxide; OD, optical density; con, control; Nor, normal.

underlying the improved IR-like state induced by 4-HIL is by acting on the TACE/TIMP3 system and consequently inhibiting TNF- $\alpha$. In addition, we explored the effect of 4-HIL on the expression of some key molecules in the insulin signaling pathway. The results demonstrated that 4-HIL suppressed the phosphorylation of both IRS-1 $\mathrm{Ser}^{307}$ and Akt Ser ${ }^{473}$, but increased the expression GLUT4 at the tested total protein (Figure 4). Therefore, activation of the insulin signaling pathway is another mechanism underlying the effects of 4-HIL on the improved IR. Finally, we investigated whether 4-HIL improved IR by influencing the differentiation of preadipocytes since PIO promotes preadipocyte differentiation and thereby alleviates IR. In our cellular model, 4-HIL did not affect the differentiation of 3T3-L1 preadipocytes (Figure 6). Moreover, nonspecific effects that might ameliorate the IR-like state, such as cytotoxic effects in adipocytes and a reduced cell number, could be excluded for 4-HIL since the viability of 3T3-L1 preadipocytes that had been exposed to 


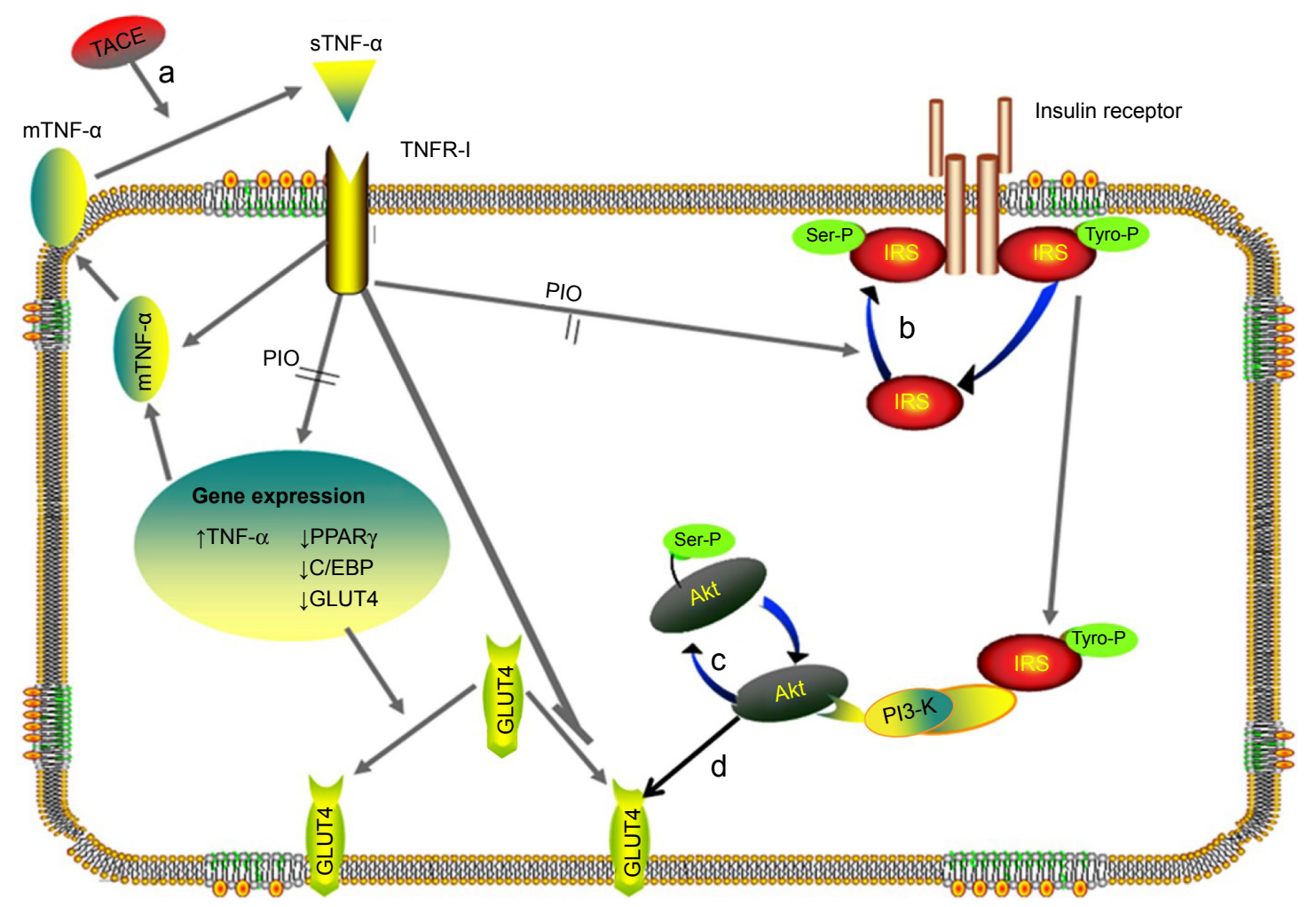

Figure 7 The targets of 4-HIL action identified in the current study.

Notes: The role of 4-HIL in the crosstalk between the inflammatory cytokine TNF- $\alpha$ and the insulin signaling pathway can be described using the following five key steps: (a) the down regulation of TNF- $\alpha$-converting enzyme (TACE) expression; (b) inhibiting the phosphorylation of IRS-I at Ser ${ }^{307}$; (c) inhibiting the phosphorylation of Akt at Ser ${ }^{473}$; (d) the upregulation of glucose transporter-4 (GLUT4) expression at the cellular membrane.

Abbreviations: 4-HIL, $(2 S, 3 R, 4 S)$-4-hydroxyisoleucine; TNF- $\alpha$, tumor necrosis factor- $\alpha$; IRS-I, insulin receptor substrate-I, PPAR, peroxisome proliferator-activated receptor; PIO, pioglitazone; sTNF- $\alpha$, soluble TNF- $\alpha$; TNFR-I, tumor necrosis factor- $\alpha$ receptor I.

high concentrations of 4-HIL for 48 hours was comparable to the viability of control cells (Figure 5).

Only one previous study has demonstrated that 4-HIL promoted the translocation of GLUT4 without changing the total amount of GLUT4 in skeletal muscle cells. ${ }^{35}$ This discrepancy might be due to differences in the cell type and the detection methods used in the two studies.

Admittedly, there remain some issues worthy of debate in the current experiment. For example, 4-HIL might inhibit substrates in addition to TNF- $\alpha$. In addition, it is possible that 4-HIL could also inhibit other members of the ADAM family ${ }^{36}$ as well as TACE. A planned follow-up study will be an in-depth analysis of the influence of 4-HIL on TACE/TIMP3 mRNA expression and TACE activity, and also confirm the present findings in vivo.

\section{Conclusion}

In conclusion, the current study demonstrated that 4-HIL could improve the IR-like state in 3T3-L1 adipocytes by 1) suppressing the activation of TNF- $\alpha$ and hence indirectly increasing glucose uptake and 2) activating insulin signal transduction and thereby directly stimulating glucose uptake.
This novel finding provides novel insights to enhance our understanding of the mode of action of 4-HIL toward improving the IR-like state.

\section{Acknowledgment}

This study was supported by the National Natural Science Foundation of China (Registered No: 81001670).

\section{Disclosure}

The authors report no conflicts of interest in this work.

\section{References}

1. Kahn SE, Hull RL, Utzschneider KM. Mechanisms linking obesity to insulin resistance and type 2 diabetes. Nature. 2006;444:840-846.

2. Ouchi N, Parker JL, Lugus JJ, Walsh K. Adipokines in inflammation and metabolic disease. Nat Rev Immunol. 2011;11:85-97.

3. Tilg H, Moschen AR. Inflammatory mechanisms in the regulation of insulin resistance. Mol Med. 2008;14:222-231.

4. Yang J, Park Y, Zhang H, et al. Feed-forward signaling of TNF-alpha and NF-kappaB via IKK-beta pathway contributes to insulin resistance and coronary arteriolar dysfunction in type 2 diabetic mice. Am J Physiol Heart Circ Physiol. 2009;296:H1850-H1858.

5. Sethi J, Hotamisligil G. The role of TNF $\alpha$ in adipocyte metabolism1. Semin Cell Dev Biol. 1999;10:19-29.

6. Gregor MF, Hotamisligil GS. Inflammatory mechanisms in obesity. Annu Rev Immunol. 2011;29:415-445. 
7. Hotamisligil GS, Shargill NS, Spiegelman BM. Adipose expression of tumor necrosis factor-alpha: direct role in obesity-linked insulin resistance. Science. 1993;259:87-91.

8. Hotamisligil GS. Inflammation and metabolic disorders. Nature. 2006;444:860-867.

9. Black RA, Rauch CT, Kozlosky CJ, et al. A metalloproteinase disintegrin that releases tumour-necrosis factor-alpha from cells. Nature. 1997;385:729-733.

10. Glass CK, Olefsky JM. Inflammation and lipid signaling in the etiology of insulin resistance. Cell Metab. 2012;15:635-645.

11. Rosen ED, Spiegelman BM. Adipocytes as regulators of energy balance and glucose homeostasis. Nature. 2006;444:847-853.

12. Stephens JM, Lee J, Pilch PF. Tumor necrosis factor-alpha-induced insulin resistance in 3T3-L1 adipocytes is accompanied by a loss of insulin receptor substrate-1 and GLUT4 expression without a loss of insulin receptor-mediated signal transduction. J Biol Chem. 1997;272: 971-976.

13. Xu H, Hirosumi J, Uysal KT, Guler AD, Hotamisligil GS. Exclusive action of transmembrane TNF alpha in adipose tissue leads to reduced adipose mass and local but not systemic insulin resistance. Endocrinology. 2002;143:1502-1511.

14. Voros G, Maquoi E, Collen D, Lijnen HR. Influence of membranebound tumor necrosis factor (TNF)-alpha on obesity and glucose metabolism. J Thromb Haemost. 2004;2:507-513.

15. Mezyk R, Bzowska M, Bereta J. Structure and functions of tumor necrosis factor-alpha converting enzyme. Acta Biochim Pol. 2003;50: 625-645.

16. Fiorentino L, Vivanti A, Cavalera M, Marzano V, Ronci M, Fabrizi M, et al. Increased tumor necrosis factor alpha-converting enzyme activity induces insulin resistance and hepatosteatosis in mice. Hepatology. 2010;51:103-110.

17. Broca $\mathrm{C}$, Breil V, Cruciani-Guglielmacci $\mathrm{C}$, et al. Insulinotropic agent ID-1101 (4-hydroxyisoleucine) activates insulin signaling in rat. $\mathrm{Am} \mathrm{J}$ Physiol Endocrinol Metab. 2004;287:E463-E471.

18. Broca C, Gross R, Petit P, et al. 4-Hydroxyisoleucine: experimental evidence of its insulinotropic and antidiabetic properties. Am J Physiol. 1999;277:E617-E623.

19. Singh AB, Tamarkar AK, Narender T, Srivastava AK. Antihyperglycaemic effect of an unusual amino acid (4-hydroxyisoleucine) in C57BL/ KsJ-db/db mice. Nat Prod Res. 2010;24:258-265.

20 Narender T, Puri A, Khaliq T, Saxena R, Bhatia G, Chandra R. 4-Hydroxyisoleucine an unusual amino acid as antidyslipidemic and antihyperglycemic agent. Bioorg Med Chem Lett. 2006;16:293-296.

21. Yu H, Wu M, Lu F-R, et al. [Effect of Trigonella foenum-graecum 4hydroxyisoleucine on high-glucose induced insulin resistance in 3T3-L1 adipocytes of mice]. Zhongguo Zhong Xi Yi Jie He Za Zhi. 2013;33:1394-1399.

22. Joo JI, Kim DH, Yun JW. Extract of Chaga mushroom (Inonotus obliquus) stimulates 3T3-L1 adipocyte differentiation. Phytother Res. 2010;24:1592-1599.
23. Tordjman KM, Leingang KA, James DE, Mueckler MM. Differential regulation of two distinct glucose transporter species expressed in 3T3-L1 adipocytes: effect of chronic insulin and tolbutamide treatment. Proc Natl Acad Sci U S A. 1989;86:7761-7765.

24. Susanti D, Amiroudine MZAM, Rezali MF, Taher M. Friedelin and lanosterol from Garcinia prainiana stimulated glucose uptake and adipocytes differentiation in 3T3-L1 adipocytes. Nat Prod Res. 2013; 27:417-424

25. Yi P, Lu F-E, Xu L-J, Chen G, Dong H, Wang K-F. Berberine reverses free-fatty-acid-induced insulin resistance in 3T3-L1 adipocytes through targeting IKKbeta. World J Gastroenterol. 2008;14:876-883.

26. Li J, Shen L, Lu F, et al. Plumbagin inhibits cell growth and potentiates apoptosis in human gastric cancer cells in vitro through the NF- $\mathrm{KB}$ signaling pathway. Acta Pharmacol Sin. 2012;33:242-249.

27. Galic S, Oakhill JS, Steinberg GR. Adipose tissue as an endocrine organ. Mol Cell Endocrinol. 2010;316:129-139.

28. Von Maltzan K, Tan W, Pruett SB. Investigation of the role of TNF- $\alpha$ converting enzyme (TACE) in the inhibition of cell surface and soluble TNF- $\alpha$ production by acute ethanol exposure. PLoS One. 2012;7: e29890.

29. Peraldi P, Xu M, Spiegelman BM. Thiazolidinediones block tumor necrosis factor-alpha-induced inhibition of insulin signaling. J Clin Invest. 1997;100:1863-1869.

30. Iwata M, Haruta T, Usui I, et al. Pioglitazone ameliorates tumor necrosis factor-alpha-induced insulin resistance by a mechanism independent of adipogenic activity of peroxisome proliferator-activated receptorgamma. Diabetes. 2001;50:1083-1092.

31. Chawla A, Nguyen KD, Goh YPS. Macrophage-mediated inflammation in metabolic disease. Nat Rev Immunol. 2011;11:738-749.

32. Shoelson SE, Lee J, Goldfine AB. Inflammation and insulin resistance. J Clin Invest. 2006;116:1793-1801.

33. Serino M, Menghini R, Fiorentino L, et al. Mice heterozygous for tumor necrosis factor-alpha converting enzyme are protected from obesity-induced insulin resistance and diabetes. Diabetes. 2007;56:2541-2546.

34. Monroy A, Kamath S, Chavez AO, et al. Impaired regulation of the TNF-alpha converting enzyme/tissue inhibitor of metalloproteinase 3 proteolytic system in skeletal muscle of obese type 2 diabetic patients: a new mechanism of insulin resistance in humans. Diabetologia. 2009; 52:2169-2181.

35. Jaiswal N, Maurya CK, Venkateswarlu K, et al. 4-Hydroxyisoleucine stimulates glucose uptake by increasing surface GLUT4 level in skeletal muscle cells via phosphatidylinositol-3-kinase-dependent pathway. Eur J Nutr. 2012;51:893-898.

36. Minematsu T, Huang L, Ibuki A, et al. Altered expression of matrix metalloproteinases and their tissue inhibitors in matured rat adipocytes in vitro. Biol Res Nurs. 2012;14:242-249.
Drug Design, Development and Therapy

\section{Publish your work in this journal}

Drug Design, Development and Therapy is an international, peerreviewed open-access journal that spans the spectrum of drug design and development through to clinical applications. Clinical outcomes, patient safety, and programs for the development and effective, safe, and sustained use of medicines are a feature of the journal, which

\section{Dovepress}

has also been accepted for indexing on PubMed Central. The manuscript management system is completely online and includes a very quick and fair peer-review system, which is all easy to use. Visit http://www.dovepress.com/testimonials.php to read real quotes from published authors. 